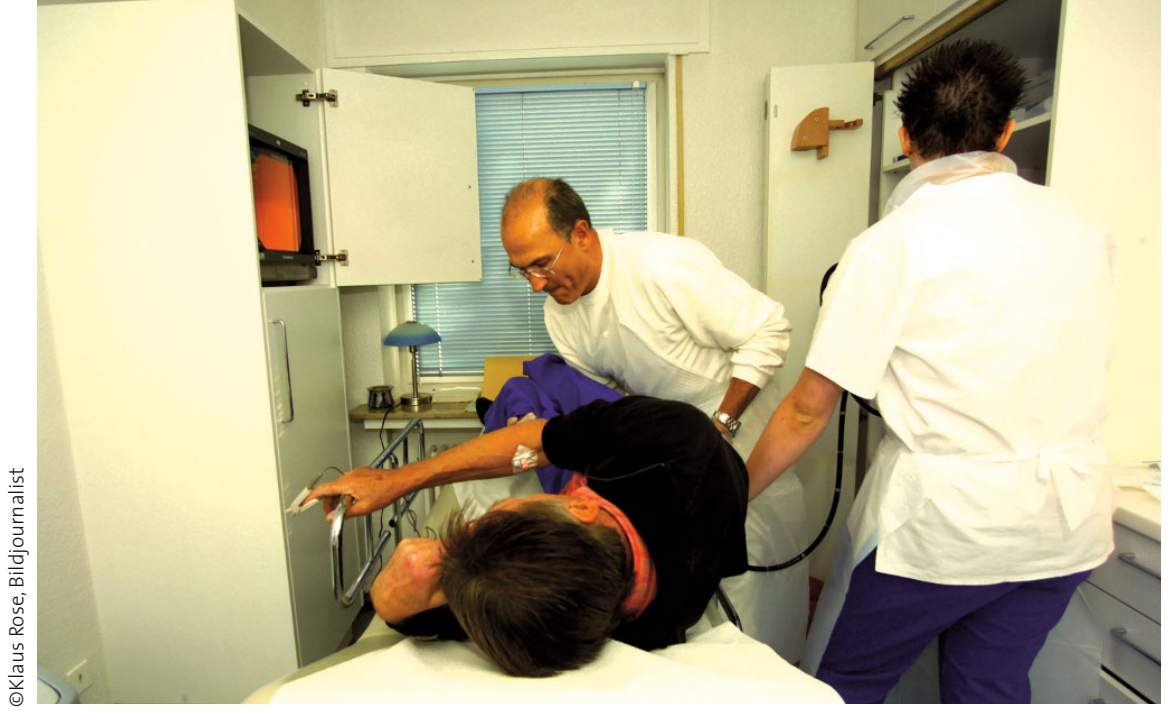

Koloskopie: Bei jedem Fünften ein relevanter Befund.

\title{
Wie effektiv und sicher ist die Vorsorgekoloskopie?
}

\section{Seit Oktober 2003 ist in Deutsch- land die Vorsorgekoloskopie für alle Personen ab dem 55. Lebensjahr etabliert. Jetzt wurden erstmals die Ergebnisse eines Onlineregisters vor- gestellt.}

- Ausgewertet wurden die Daten von 269000 Vorsorgekoloskopien aus 280 gastroenterologischen Praxen. Bei $15,6 \%$ der Untersuchten wurden tubuläre Adenome, bei 3,7\% villöse/tubulovillöse Adenome, und bei $0,8 \%$ invasive Karzinome detektiert. Fortgeschrittene Adenome definiert durch einen Durchmesser von mindestens $10 \mathrm{~mm}$, eine villöse oder tubulovillöse Komponente oder das Vorhandensein einer hochgradigen Dysplasie, fanden sich bei $7,1 \%$ der untersuchten Personen. 95\% der Polypen wurden unmittelbar während der Vorsorgekoloskopie abgetragen.

Bei den entdeckten Karzinomen handelte es sich in $43 \%$ um ein Stadium I, in $27 \%$ um ein Stadium II. Die Komplikationsrate der Koloskopie war insgesamt niedrig: Kardiopulmonale Komplikationen traten bei $0,1 \%$ der Personen, Blutungen nach Polypektomie bei $0,8 \%$ der Fälle auf, wobei die Mehrzahl der Blutungen endoskopisch gestillt werden konnte. Perforationen wurden bei $0,02 \%$ der Koloskopien und $0,09 \%$ der Polypektomien gemeldet, jedoch kein Todesfall.

\section{Kommentar}

Diese Daten belegen erneut, dass bei jedem Fünften, der sich der Vorsorgekoloskopie unterzieht, ein prognostisch relevanter Befund, nämlich ein Polyp oder ein Karzinom, entdeckt wird. Die überwiegende Mehrzahl der entdeckten Karzinome war in einem niedrigen Stadium, was letztendlich die Prognose betroffener Patienten verbessern dürfte. Auch erwies sich das Verfahren insgesamt als sicher. Doch ein Problem, das nicht im Rahmen dieses Registers erfasst wurde, sind die Intervallkarzinome. Darunter versteht man Dickdarmkarzinome, die bei Einhaltung der empfohlenen Kontrollintervalle eigentlich nicht auftreten dürften, über die aber immer häufiger berichtet wird. $\mathrm{Ob}$ es sich dabei um Karzinome mit besonderen biologischen Charakteristika oder um übersehene oder fehlinterpretierte Befunde handelt, darüber wird intensiv diskutiert. Doch der gastroenterologische Alltag zeigt, dass heute weniger das Auflösungsvermögen des Untersuchers und der eingesetzten Endoskope als vielmehr die Qualität der Vorbereitung der limitierende Faktor für das Erkennen von polypösen bzw. tumorösen Veränderungen darstellt.

P. StIEFELHAGEN =

- B. Bokemeyer et al.

Screening colonoscopy for colorectal cancer prevention; results from a German online registry on 269000 cases. Eur. J. Gastroenterol. Hepatol. 21 (2009) 650-655 